\title{
A Salvage Operation for Total Penis Amputation Due to Circumcision
}

\author{
Bilsev Ince, Ayse Ozlem Gundeslioglu \\ Department of Plastic and Reconstructive and Aesthetic Surgery, Necmettin Erbakan University, Faculty of Meram Medicine, Konya, Turkey
}

Circumcision is one of the most common rituals in Jewish and Islamic cultures. It may also be performed for phimosis correction or the treatment of recurrent balanitis. Although circumcision is considered to be a technically easy and safe surgical procedure with no significant risk, it may lead to severe complications such as necrotizing fasciitis or total penis amputation. In this report, we present a case of penis amputation at two levels occurring with third-degree burns due to electrocautery during circumcision. Although penile replantation was attempted, it was unsuccessful due to burn damage to the veins. After restoration of the functional structures, the penis was buried in the inguinal area by reepithelization to maintain blood circulation. The recovery of the penis was successful. This case is presented as a novel example of groin flap surgery to achieve a functionally and aesthetically acceptable outcome in a salvage operation for a penis with significant traumatic injury, which has not been previously reported in the literature.

Keywords Penis / Circumcision / Amputation
Correspondence: Bilsev Ince Department of Plastic and Reconstructive and Aesthetic Surgery, Necmettin Erbakan University, Faculty of Meram Medicine, 42080 Meram, Konya, Turkey

Tel: +90-332-223-60-00-7791

Fax: +90-332-323-61-81

E-mail: bilsevince@yahoo.com

No potential conflict of interest relevant to this article was reported.

\section{INTRODUCTION}

Circumcision is one of the most common rituals in Jewish and Islamic cultures. It may also be performed for phimosis correction or the treatment of recurrent balanitis [1]. Although circumcision is considered to be a technically simple and safe surgical procedure with no significant risk, it may occasionally lead to severe complications such as necrotizing fasciitis or total penis amputation [2-4].

In this report, we present a case of penis amputation at two levels occurring with third-degree burns due to electrocautery during circumcision. Although penile replantation was attempted, it was unsuccessful due to burn damage to the veins. After restoration of the functional structures, the penis was buried in the inguinal area by reepithelization to maintain blood circulation. The recovery of the penis was successful. This case is presented as a novel example of groin flap surgery to achieve a functionally and aesthetically acceptable outcome in a salvage operation for a penis with significant traumatic injury, which has not been previously reported in the literature.

\section{CASE}

An 8-year-old child with penis amputation due to circumcision was admitted to Emergency Services. A physical examination demonstrated that the penis was completely amputated from the glans penis (Fig. 1). In addition, a second partial amputation was present in the skin of the corpus penis with a third-degree burn 


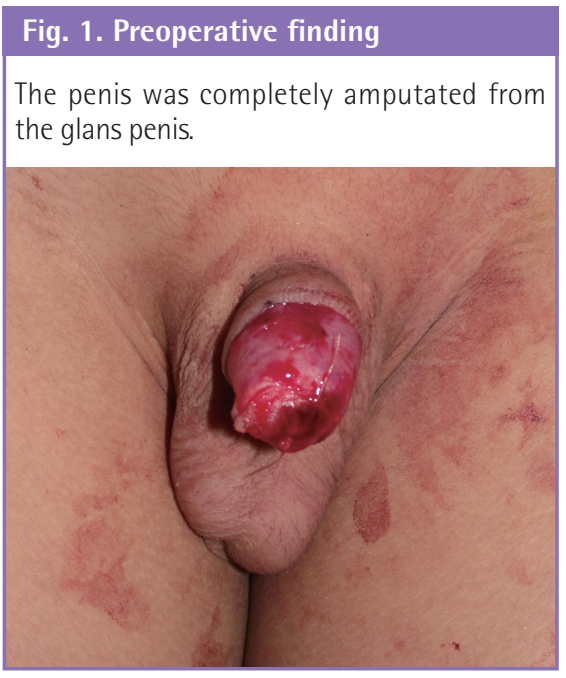

Fig. 4. The penis was removed from the inguinal skin

Scar and minimal tissue loss were present on the penis.

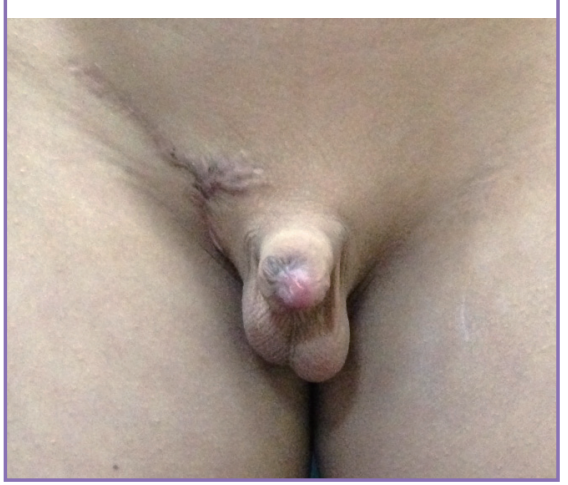

\section{Fig. 2. A second partial amputation}

A second partial amputation was present in the corpus penis with third-degree burns to an area including the prepuce.

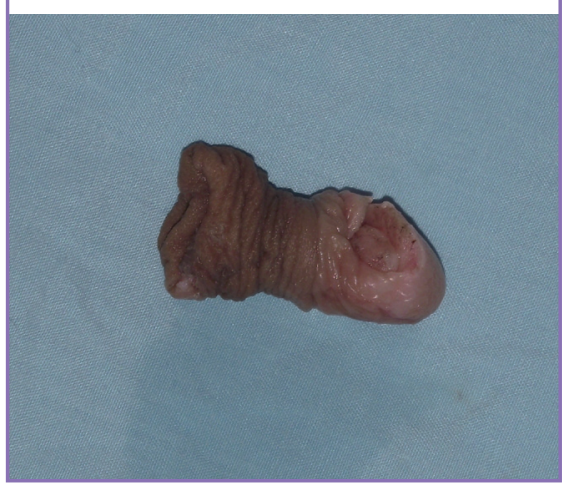

\section{Fig. 5. Good voiding function}

Good voiding function of the patient is seen on the follow-up at 6 months.

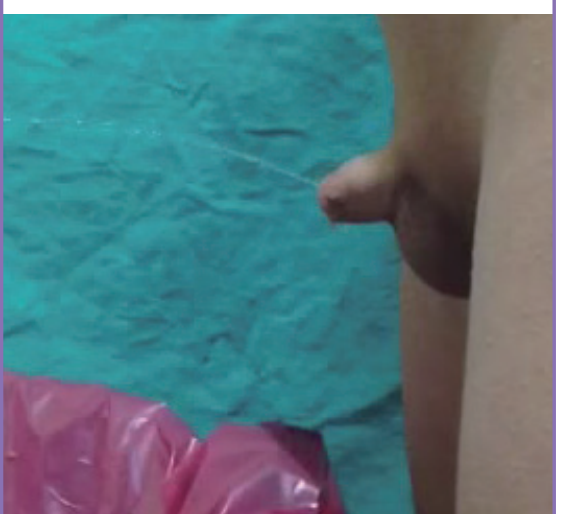

Fig. 3. The penis was buried under the inguinal skin

The catheter was removed from the hole and the penis was buried under the inguinal area.

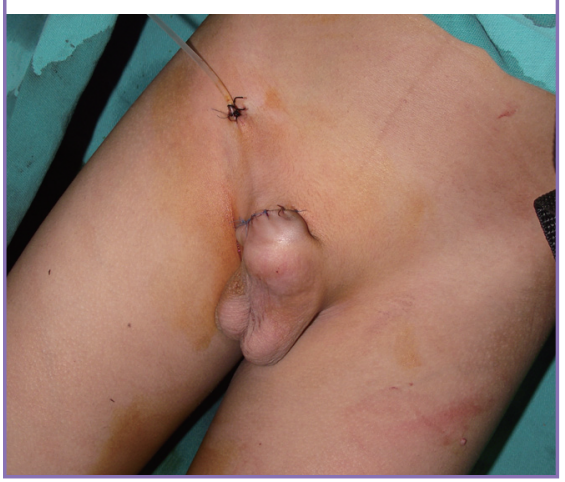

Fig. 6. Good erectile function

Good erectile function of the patient is seen on the follow-up at 6 months.

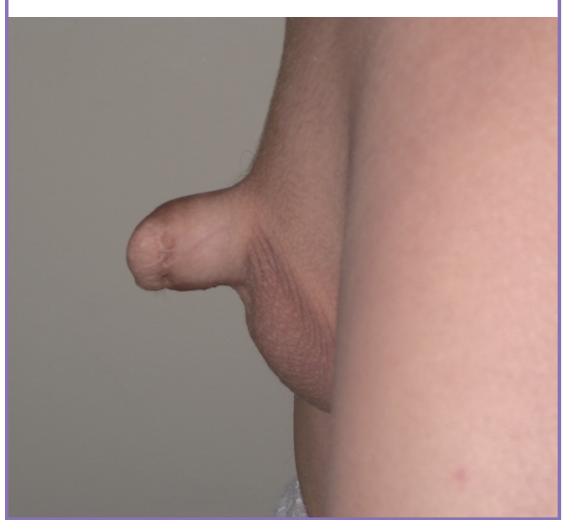

including the prepuce (Fig. 2). The patient history revealed that he was circumcised by an inexperienced person at home under local anesthesia using electrocautery one hour before admission to the emergency service.

The patient was taken immediately to the operating room and was operated on under general anesthesia. Escharotomy was performed at the areas with third-degree burns. The urethra, corpus cavernosum, and septum were repaired by inserting an 8 F Foley catheter into the urethra. The deep dorsal penile artery was explored and irrigated with heparinized saline. It was then repaired by end-to-end anastomosis with $10-0$ polypropylene suture material under a surgical microscope, and Buck's fascia was also repaired. However, because the burned tissues were too damaged to repair the veins, complete blood circulation of the penis could not be achieved, and venous congestion occurred while the patient was still in the operating room. The amputated glans penis and the penis corpus skin were deepithelized, and a hole was created in the inguinal skin for a Foley catheter. The catheter was removed from the hole, and the penis was buried underneath the inguinal area (Fig. 3). A dressing with antibiotic ointment was applied daily. Three weeks later, the penis was removed from the inguinal skin, and the glans and the corpus of the penis appeared healthy.

Although scar and minimal tissue loss were present on the penis (Fig. 4), good voiding (Fig. 5) and erection were obtained by the 6-month follow-up (Fig. 6). In addition, sensation had recovered after 6 months.

\section{DISCUSSION}

This case study demonstrated that a penis amputated on two different levels with electrocautery and a wide burn injury was able to be recovered successfully with less damage than expected.

The success of replantation following penis amputation is associated with the level of the incision, the incision type (crush or sharp incision), and the ischemia time. In cases of a simple one- 
level proximal penis amputation, replantation is usually successful and should be attempted if the amputated piece is preserved properly and the timing of the repair is appropriate [5]. It is important to repair the vein for tissue survival [5]. In this amputation case, which was accompanied by a third-degree burn due to electrocautery, vein repair was not possible, but blood circulation was achieved by burying the amputated piece underneath the inguinal area.

However, in some cases, vessels may not be found for repair or could be impossible to repair in cases of distal amputations. In Thailand, skin loss was observed in 12 of 14 replantations, and following the primary suture of the penis as a graft, loss was observed in 6 of 14 replantations performed without microsurgery [6]. Hyperbaric oxygen treatment was attempted in a patient with partial glans amputation following the development of necrosis seven days after primary repair. The necrosis had improved at the second level of amputation within four weeks, and there was no functional loss [7].

In cases of penis replantation, the functional units of the penis are repaired, and the penis may regain its former esthetic appearance. Erectile function and sensory innervation are regained within an average of one year after penis replantation $[4,8]$. In the present case, the early return of sensory innervation and erectile function was linked to patient age.

In routine groin flap surgery, the tissue requiring nourishment is buried underneath the inguinal area for three weeks, and the blood circulation of the flap is achieved by the donor. After three weeks, when the connection between the donor and the receiving sites is complete, collateral vessels will have formed within the recipient tissue for nourishment [9]. In addition, in routine neurosurgery, the survival of cranial pieces that cannot be repaired immediately is ensured by burying them under the inguinal area. The buried cranium can be removed for repair once the reconstruction is planned [10]. In our case, the tissue lacking blood circulation was introduced as a composite graft through reepithelization, and blood circulation and the survival of the amputated piece were successfully achieved.

If replantation cannot be performed following total penis amputation, using free flaps may be an option for late repair of the tissue. When early repair is not possible, free flaps are preferred, and the functions of the amputated part can be partially regained. The total amputation of the penis as a complication of circumcision and its successful replantation have been reported in the literature [11-13]. The first choice should be replantation in the treatment of total penis amputation. However, if replantation cannot be performed for amputations on multiple levels and/ or the amputation is accompanied by an electrocautery-induced burn, burying the amputated part underneath the inguinal area is an alternative choice for treatment. Our case is unique because the amputation occurred at multiple levels and was accompanied by an electrocautery-induced burn. A functionally and aesthetically acceptable salvage operation for a penis with similar injuries has not been previously reported.

In conclusion, circumcision is a surgical procedure that should be performed by surgeons under sterile conditions in a health center. When the appropriate conditions for circumcision are not provided, complications may occur that lead to total amputation. For distal penis amputations in which the repair cannot be achieved by replantation, burying the penis underneath the inguinal area may be an alternative salvage operation to replantation.

\section{REFERENCES}

1. Rickwood AM. Medical indications for circumcision. BJU Int 1999;83 Suppl 1:45-51.

2. Gecit I, Benli E. Bolgemizdeki sunnet komplikasyonları. Van Tip Dergisi 2011;18:155-8.

3. Gee WF, Ansell JS. Neonatal circumcision: a ten-year overview: with comparison of the Gomco clamp and the Plastibell device. Pediatrics 1976;58:824-7.

4. Gyan S, Sushma S, Maneesh S, et al. Successful microsurgical penile replantation following self amputation in a schizophrenic patient. Indian J Urol 2010;26:434-7.

5. Tazi MF, Ahallal Y, Khallouk A, et al. Spectacularly successful microsurgical penile replantation in an assaulted patient: one case report. Case Rep Urol 2011;2011:865489.

6. Bhanganada K, Chayavatana T, Pongnumkul C, et al. Surgical management of an epidemic of penile amputations in Siam. Am J Surg 1983;146:376-82.

7. Faydaci G, Ugur K, Osman C, et al. Amputation of glans penis: a rare circumcision complication and successful management with primary anastomosis and hyperbaric oxygen therapy. Korean J Urol 2011;52:147-9.

8. Cohen BE, May JW Jr, Daly JS, et al. Successful clinical replantation of an amputated penis by microneurovascular repair. Case report. Plast Reconstr Surg 1977;59:276-80.

9. Acarturk S, Ozmen E, Kaya Y, et al. El ve onkol defektlerinin kasık flebi ile onarımı. Turk Plast Surg 1982;4:17-9.

10. Ergin N, Kadioglu HH. Reconstruction of cranial defects with acrylic, otolog and homolog bone grafts. Turk Neurosurg 1996;6:24-7.

11. Yilmaz AF, Sarikaya S, Yildiz S, et al. Rare complication of circumcision: penile amputation and reattachment. Eur Urol 1993;23:423-4.

12. Lee SY, Yang CS, Myung SC. Replantation of a completely 
amputated penis, which occurred in a child during circumcision. Korean J Urol 2005;46:1360-2.

13. Ozkan S, Gurpinar T. A serious circumcision complication: penile shaft amputation and a new reattachment technique with a successful outcome. J Urol 1997;158:1946-7. 